\title{
Intermittent saltation drives Mars-like aeolian activity on Titan
}

\author{
F. Comola ${ }^{1, \dagger}$, J. F. Kok ${ }^{1}$, J. M. Lora ${ }^{2}$, K. Cohanim ${ }^{1}$, X. Yu ${ }^{3}$, C. He ${ }^{4}$, P. McGuiggan ${ }^{4}$, S. M. \\ Hörst $^{4}$, and F. Turney ${ }^{1}$ \\ ${ }^{1}$ Department of Atmospheric and Oceanic Sciences, University of California, Los Angeles, USA. \\ ${ }^{2}$ Department of Earth and Planetary Sciences, Yale University, New Haven, USA. \\ ${ }^{3}$ Department of Earth and Planetary Sciences, University of California, Santa Cruz, USA. \\ ${ }^{4}$ Department of Earth and Planetary Sciences, Johns Hopkins University, Baltimore, USA. \\ ${ }^{\dagger}$ Corresponding author: Francesco Comola, francesco.comola@gmail.com, Twitter
}

This paper is a non-peer reviewed preprint submitted to EarthArXiv.

\begin{abstract}
Titan, the largest moon of Saturn, is characterized by gigantic linear dunes and an active dust cycle. Much like on Earth, these and other aeolian processes are caused by the wind-driven mobilization of surface grains, known as saltation. To date, very little is known about the conditions that allow for the occurrence of saltation on Titan. In fact, Titan saltation may be fundamentally different from Earth saltation given the denser atmosphere, the lower gravity, and the cohesion of its surface grains. Here, we draw on experiments, theory, and modeling to progress towards a comprehensive understanding of saltation on Titan. We find that aerodynamic lifting of surface grains requires strong wind speeds due to the high cohesion of the grains. However, saltation may be sustained through granular splash at wind speeds much smaller than those required to initiate grain motion. This suggests that most saltation transport on Titan is intermittent rather than continuous. We account for these insights by proposing a saltation mass flux parameterization specific for Titan conditions that accounts for transport intermittency, and use it to quantify yearly sediment transport with a general circulation model. The results show that Titan's prevailing atmospheric circulation is capable of generating highly intermittent yet significant saltation, yielding yearly transport rates similar to those on the most active dunes of Mars. Furthermore, we find that accounting for surface topography might be critical to answering open questions related to Titan's landscape evolution, including the formation of linear dunes in opposite direction to the prevailing circulation.
\end{abstract}

\section{Significance statement}

The Cassini-Huygens mission has revealed that Titan's landscape evolution and dust cycle may be controlled by the wind-driven transport of surface grains, known as saltation. It is still unclear, however, how saltation can occur on Titan despite the weak winds and the stickiness of the surface grains. Using a combination of experiments, theory, and modeling, we find that, like saltation on Mars, saltation on Titan can be sustained at much lower wind speeds than those required to lift grains from the surface. Accordingly, the prevailing weak winds on Titan may be capable of driving intermittent yet significant saltation, with transport rates comparable to those observed on the most active dunes on Mars.

\section{Introduction}

The Cassini-Huygens mission has revealed that Titan's low latitude surface presents a variety of landforms Lorenz et al., 2006, Lopes et al., 2019, MacKenzie et al., 2021, including gigantic linear dunes similar in shape to those of the Namib desert [Radebaugh et al., 2008, 2010]. Analyses of Cassini spectral data, combined with atmospheric and radiative transfer modeling, have further revealed that Titan presents an active dust cycle Charnay et al. 2015, Rodriguez et al. 2018. This observational evidence suggests that, 
much like on Earth, Titan dunes actively evolve by an aeolian, or wind-driven, transport process known as saltation: after being lifted and accelerated by the wind, surface grains hop along the granular bed, rebounding and splashing other grains into the airflow Kok et al. 2012, Pähtz et al., 2020.

Titan's sand grains are not made of silicates as on Earth but mainly of solid organics precipitated from the atmosphere [Lorenz, 2014. Even though their physical properties are not precisely known, previous studies have suggested that these organic grains could be less dense and more cohesive than quartz sand Imanaka et al., 2012, Hörst and Tolbert, 2013, He et al., 2017, Méndez-Harper et al., 2017, Yu et al., 2017, $2020 \mathrm{a}$ b. This, in combination with Titan's denser atmosphere and reduced gravity, leads to fundamental differences in dune formation on Earth and Titan. One of the main, yet poorly understood differences is that Titan's dunes appear to be shaped by surface winds opposite in direction to the prevailing atmospheric circulation Tokano, 2008, McDonald et al., 2016, Ewing et al., 2015. A commonly accepted explanation is that the threshold wind speed required to initiate saltation, the so-called fluid threshold, lies above the speed of the prevailing easterly winds Burr et al. 2015 but below the speed of stronger westerlies generated by equatorial methane storms at equinox Charnay et al. 2015]. This is based on the consensus that particle lifting on Titan is done primarily by aerodynamic forces and that transport cannot be sustained below the fluid threshold [Kok et al., 2012]. Recent studies, however, have suggested that saltation of cohesive grains, such as those on Titan, can be sustained through rebound and granular splash at much lower wind speeds than those required to initiate grain motion Comola et al., 2019a, Pähtz et al., 2021. The role of the fluid threshold in Titan's dust cycle and landscape evolution may therefore be less relevant than previously thought.

Here, we aim to shed light onto aeolian transport processes on Titan through a combination of laboratory experiments, theory, and numerical modeling. For this purpose, we propose novel parameterizations for the aerodynamic entrainment and granular splash processes that account for the effect of cohesive forces among surface grains. We specify the key physical parameters of these parameterizations, namely grain density, elasticity, and cohesion, based on recent experimental investigations Yu et al., 2017 and test their performance against the results of a discrete element model. We then account for the proposed entrainment parameterizations in the saltation model COMSALT Kok and Renno, 2009 to investigate how sediment mass flux scales with friction velocity on Titan. We finally include the mass flux parameterization in the general circulation model TAM Lora et al., 2015, 2019] to quantify yearly sediment transport rates and drift directions on Titan. We find that Titan's prevailing circulation drives intermittent sediment transport of the order what is found in mobile Martian dune fields.

\section{Fluid threshold on Titan}

A correct estimation of the fluid threshold is essential to understand the conditions that allow for aerodynamic entrainment of grains on Titan. To estimate the fluid threshold, we use the well-known parameterization Shao and Lu, 2000

$$
u_{*, f t}=\sqrt{A_{N}\left(\frac{\rho_{p}}{\rho_{f}} g d+\frac{\gamma}{\rho_{f} d}\right)},
$$

where $A_{N}=0.0123$ is an empirical dimensionless parameter, $g \approx 1.35 \mathrm{~m} \mathrm{~s}^{-2}$ is the gravitational constant, $d$ is the particle diameter, $\rho_{f} \approx 5.2 \mathrm{~kg} \mathrm{~m}^{-3}$ is the air density, $\rho_{p} \approx 950 \pm 450 \mathrm{~kg} \mathrm{~m}^{-3}$ is the particle density (uncertainty estimations throughout the paper refer to standard errors), and $\gamma$ is a cohesion coefficient. Cohesion is related to the intrinsic stickiness of the material (the surface energy), the particle shape and roughness, the stiffness of the contacting grains, and the moisture conditions [Israelachvili, 1986]. It is usually assumed that $\gamma \propto \beta=F_{\phi} / d$, that is, the ratio between the cohesive force $F_{\phi}$ and the particle size $d$. It is important to note that $\beta$ represents the average cohesive force between grains, whereas $\gamma$ represents the cohesive force acting on the grains that are more readily lifted by the wind. Because of this discrepancy, the 
proportionality constant between $\gamma$ and $\beta$ is generally unknown. We therefore estimate $\gamma$ for Titan grains by assuming that the ratio of $\gamma$ and $\beta$ is equal on Earth and Titan, that is,

$$
\frac{\gamma_{T}}{\beta_{T}}=\frac{\gamma_{E}}{\beta_{E}} .
$$

Measurements of fluid threshold for quartz sand suggest that $\gamma_{E} \approx 0.33 \pm 0.17 \mathrm{mN} \mathrm{m}^{-1}$ Shao and Lu, 2000. Furthermore, laboratory measurements of the cohesive forces for quartz sand and Titan-analog grains, known as tholins, suggest that $\beta_{E} \approx 1.2 \mathrm{mN} \mathrm{m}^{-1}$ [Corn, 1961] and $\beta_{T} \approx 27 \pm 20 \mathrm{mN} \mathrm{m}^{-1}$ [Yu et al., 2017]. Based on equation (2) and accounting for error propagation, we estimate that $\gamma_{T} \approx 7.3 \pm 6.7 \mathrm{mN} \mathrm{m}^{-1}$. We use this range of $\gamma_{T}$ in equation (1) to estimate the variation in fluid threshold with particle size (Figure 1). The results indicate that the minimum shear velocity required to lift a grain on Titan (red curve in Figure 1) is $u_{*, f t} \approx 0.12 \mathrm{~m} \mathrm{~s}^{-1}$, which is approximately three times larger than expected if cohesive forces among organic grains on Titan were equal to those among sand particles on Earth (green curve in Figure 1). Furthermore, this minimum value corresponds to a particle size $d \approx 2 \mathrm{~mm}$, meaning that the particles that are easiest to lift on Titan are roughly one order of magnitude larger than sand particles that are easiest to lift on Earth (blue curve in Figure 1). Critically, we find that previous measurements carried out in a wind tunnel with environmental conditions similar to those on Titan (black circles in Figure 1) may have significantly underestimated the fluid threshold and the size of the more mobile grains on Titan due to the low cohesion of the sediments used for the experiments Burr et al., 2015, Yu et al., 2017].

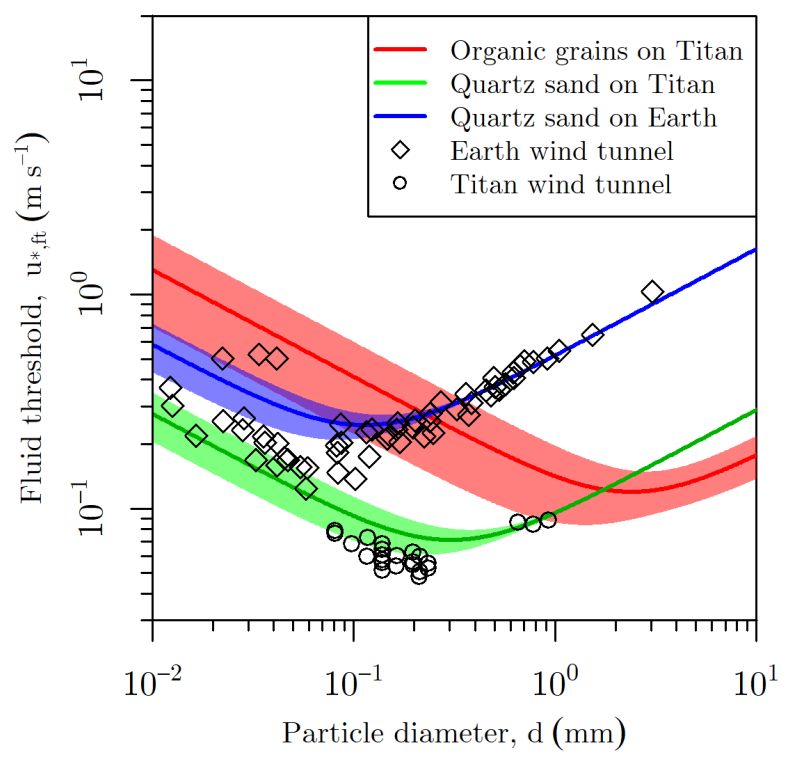

Figure 1: Variation in the fluid threshold with particle size on Earth and Titan. The red curve refers to organic grains on Titan, the blue one refers to quartz grains on Earth, and the green one is the hypothetical fluid threshold of quartz grains on Titan. The shaded areas indicate standard errors, obtained by propagating the uncertainties in the cohesion coefficient $\gamma$ and particle density $\rho_{p}$. Black diamond markers indicate wind tunnel measurements of the fluid threshold in Earth conditions [Bagnold, 1937, Chepil, 1945. Zingg, 1953, Iversen et al., 1976, Fletcher, 1976. Black circles indicate fluid threshold measurements carried out in the Titan wind tunnel Burr et al.|2015 for sediments with weaker cohesive bonds than organic grains on Titan (silica sand, basaltic sand, glass spheres, walnut shells). 


\section{Impact threshold on Titan}

Our analyses have so far suggested that the fluid threshold on Titan may be significantly higher than previously thought due to the high cohesion of surface grains. We now investigate the effect of cohesion on the minimum wind speed required to sustain saltation through the granular splash process, the so-called impact threshold $u_{*, i t}$ Pähtz and Durán, 2018. Granular splash is a complex and highly stochastic process controlled by interparticle collisions and cohesive bonds among neighboring grains. To predict the mean velocity of the splashed grains $\left\langle v_{s}\right\rangle$, we extend an expression for loose granular materials Kok and Renno, 2009 with an additional term that accounts for the effect of cohesion (see supporting information section S1 for the analytical derivation)

$$
\frac{\left\langle v_{s}\right\rangle}{\sqrt{g d}}=\frac{\mu}{a}\left[1-\exp \left(-a \frac{v_{i}}{\sqrt{g d}}\right)\right]+b \sqrt{\frac{2(1-\delta) \phi}{m g d}} .
$$

In equation (3), $\phi$ is the elastic energy released upon the breaking of cohesive bonds, and $\delta \approx 0.3$ is the fraction of elastic energy dissipated. The elastic energy $\phi$ is a function of the cohesive force $F_{\phi}$ and the effective bond elastic modulus $E$, which we estimate from experiments on tholin particles Yu et al. 2017 (see supporting information section S1 and Table S2). Further, $\mu \approx 0.15$ is the average fraction of impacting momentum spent on splashing surface particles Kok and Renno, 2009]. The proportionality coefficients $a \approx 0.03$ and $b \approx 1.2$, which scale the contributions of collisional and cohesive forces to the ejection velocity, are assigned based on literature values Kok and Renno, 2009 and by fitting data from discrete element simulations of splash process over cohesive surfaces (see supporting information section S1).

To estimate the mean number of splashed grains $\left\langle N_{s}\right\rangle$, we adopt a splash model derived from the energy and momentum conservation equations Comola and Lehning, 2017]. This model was shown to be in good agreement with a variety of experimental results, including granular splash data of cohesive snow and ice grains. For a granular bed of uniform spherical grains, the average number of splashed grains predicted by the energy conservation equation equals

$$
\left\langle N_{e}\right\rangle=\frac{\left(1-\mathrm{P}_{r} \epsilon_{r}-\epsilon_{f}\right) v_{i}^{2}}{2\left\langle v_{s}\right\rangle^{2}+\frac{12 \delta \phi}{\pi \rho_{p} d^{3}}},
$$

where $\epsilon_{r}$ is the fraction of impact energy retained by the rebounding grain, $\mathrm{P}_{r}$ is the probability of rebound Anderson and Haff, 1991, Andreotti, 2004, and $\epsilon_{f}$ is the fraction of energy dissipated to the bed. Furthermore, the average number of splashed grains predicted by the horizontal momentum conservation equation equals

$$
\left\langle N_{m}\right\rangle=\frac{\left(1-\mathrm{P}_{r} \mu_{r}-\mu_{f}\right) v_{i} \cos \alpha_{i}}{\left\langle v_{s}\right\rangle\left\langle\cos \alpha_{s}\right\rangle\left\langle\cos \beta_{s}\right\rangle}
$$

where $\mu_{r}$ is the fraction of momentum retained by the rebounding grain, $\mu_{f}$ is the fraction of momentum lost to the bed, $\alpha_{i}$ is the vertical impact angle, $\cos \alpha_{s}$ is the cosine of the vertical splash angle, and $\cos \beta_{s}$ the cosine of the horizontal splash angle. The values of all parameters in equations (4) and (5) are assigned based on experimental measurements Willetts and Rice, 1986, 1989, Rice et al., 1995, 1996, Nalpanis et al., 1993, Ammi et al., 2009 (see Table S1 in the supporting information). Following previous approaches Kok and Renno, 2009, Comola and Lehning, 2017], we take the number of splashed grains as $\left\langle N_{s}\right\rangle=\min \left(\left\langle N_{e}\right\rangle,\left\langle N_{m}\right\rangle\right)$, to represent the transition from a momentum-limited to an energy-limited splash process. We discuss the generalizations of equations (3)-(5) for mixed-sized granular beds in the supporting information (section $\mathrm{S} 1)$. 
We test the predictions of equations (3)-(5) against the results of a discrete element model that was previously used to investigate the role of cohesion in the granular splash process [Comola et al., 2019a (see the supporting information section S2 for details on the model equations). Equations (3)-(5) closely reproduce the variation in velocity and number of splashed grains with cohesion predicted by the discrete element simulations for different combinations of grain size and impact velocity (Figure 2). The results suggest that the splash process is weakly sensitive to cohesion when the energy released by cohesive bonds is small compared to the gravitational potential energy $\left(\phi / m g d \lesssim 10^{-1}\right)$. Conversely, cohesion exerts a relevant control on the splash process for larger values of $\phi / m g d$ by increasing the mean splash velocity (Figures 2a and 2c) and decreasing the number of splashed grains (Figures $2 \mathrm{~b}$ and $2 \mathrm{~d}$ ). The physical reason for these results is that stronger cohesive bonds, albeit more unyielding, release a larger amount of elastic energy upon breaking, thereby increasing the grain ejection velocity Comola et al., 2019a. We find that cohesive forces have a small impact on the granular splash of organic grains on Titan. The cohesive energy $\phi$, estimated from the experimental measurements Yu et al. 2017, is in fact barely sufficient to affect the granular splash process of particles of size $d=0.25 \mathrm{~mm}$ (gray areas in Figures $2 \mathrm{a}$ and $2 \mathrm{~b}$ ). Cohesion is even less relevant for the granular splash of coarser grains of size $d=2.5 \mathrm{~mm}$ (gray areas in Figures 2c and 2d), which are primarily splashed through chains of interparticle collisions, similar to how sand grains on Earth are splashed (black markers in Figures 2a and 2b) Crassous et al. 2007.
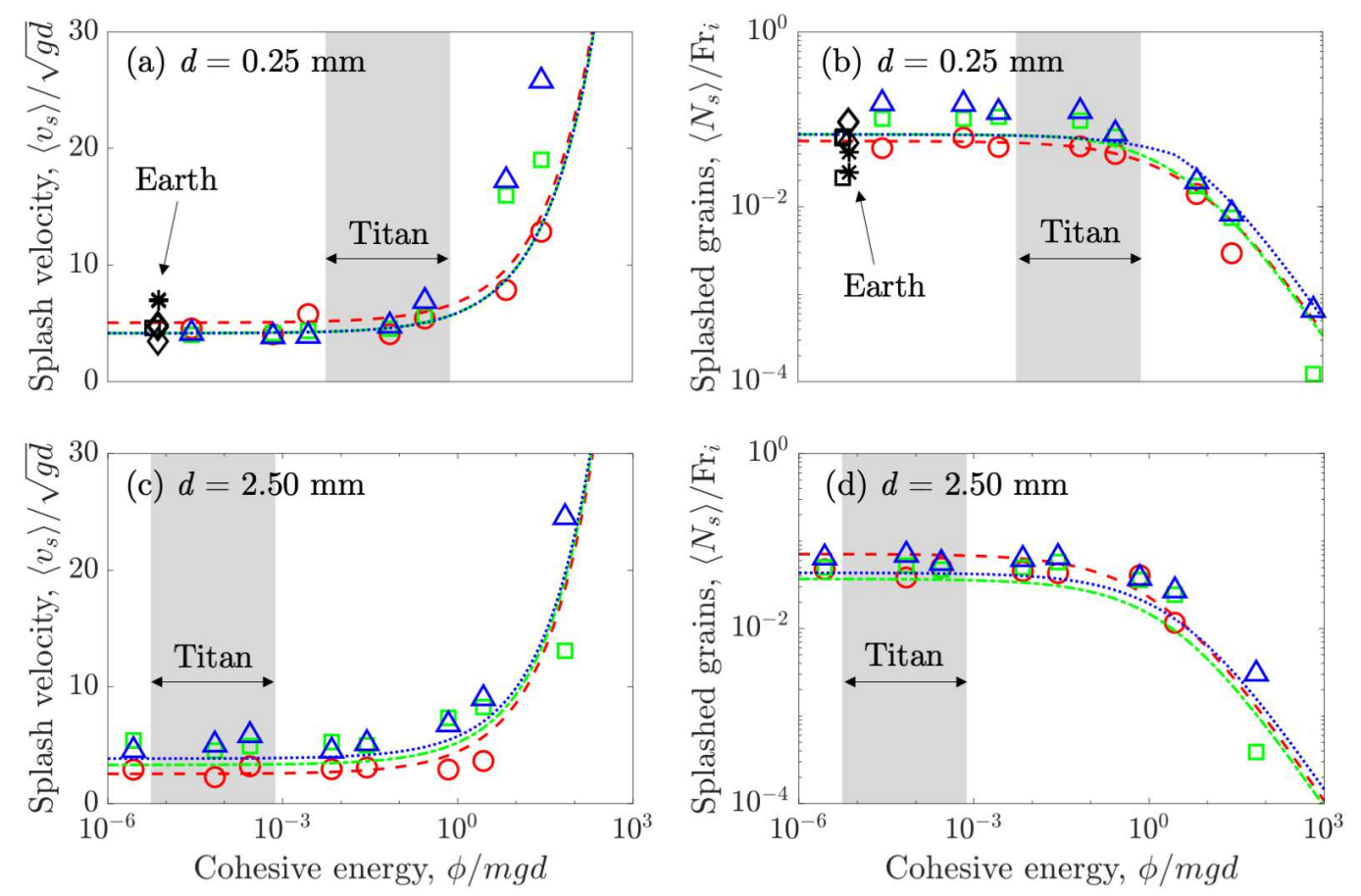

Figure 2: Variation in velocity and number of splashed grains with cohesion. Colored lines refer to analytical results of equations (3)-(5), colored markers refer to discrete element simulations, and black markers refer to experimental results. Marker and line colors indicate different values of impact velocity $v_{i}$, with red color indicating $v_{i}=1 \mathrm{~m} \mathrm{~s}^{-1}$, green color $v_{i}=3 \mathrm{~m} \mathrm{~s}^{-1}$, and blue color $v_{i}=5 \mathrm{~m} \mathrm{~s}^{-1}$. (a) Velocity and (b) number of splashed grains from a monodisperse granular bed with particle size $d=0.25 \mathrm{~mm}$. The number of splashed grains is normalized by the Froude number of the impacting grain $\operatorname{Fr}_{i}=v_{i} / \sqrt{g d}$. Black markers indicate experimental results for sand particles of similar size $(\langle d\rangle \approx 0.3 \mathrm{~mm})$ Willetts and Rice, 1985, 1986, Rice et al., 1995. Willetts and Rice, 1989, Rice et al., 1996, Gordon and McKenna-Neuman, 2011. (c) Velocity and (d) number of splashed grains from a monodisperse granular bed with particle size $d=2.5 \mathrm{~mm}$. The shaded gray areas indicate the estimated range of cohesion for organic grains on Titan. 
To investigate the effect of cohesion on the impact threshold $u_{*, i t}$ on Titan, we implement equations (3)(5) in the comprehensive saltation model COMSALT Kok and Renno, 2009, Kok, 2010a b and simulate Titan saltation for a wide range of cohesive forces (see the supporting information sections S3 and S4 for the implementation details). Critically, we find that, even though cohesive forces greatly increase the fluid threshold on Titan, they only slightly affect the impact threshold of grains larger than $0.1 \mathrm{~mm}$ (blue lines in Figure 3), and only moderately increase the impact threshold of smaller grains. Most importantly, the minimum impact threshold $u_{*, i t} \approx 0.03 \mathrm{~m} \mathrm{~s}^{-1}$ is a factor of four smaller than the minimum fluid threshold, suggesting that Titan saltation may be sustained at wind speeds much smaller than those required to initiate it. Furthermore, the minimum impact threshold corresponds to a particle size $d \approx 0.1 \mathrm{~mm}$, which is one order of magnitude smaller than the size of particles most easily lifted by aerodynamic forces.

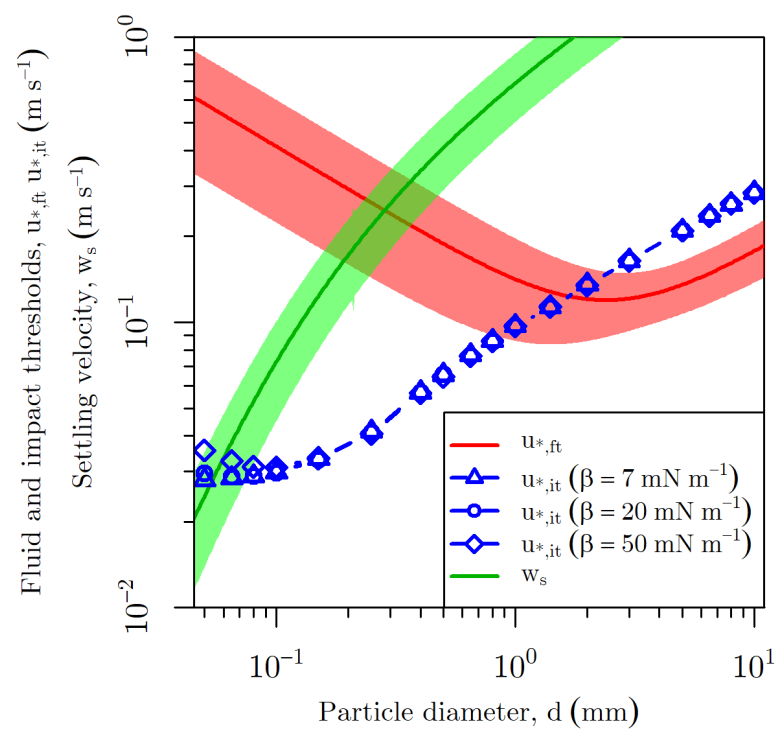

Figure 3: Variation in fluid threshold, impact threshold, and settling velocity of grains on Titan. The fluid threshold $u_{*, f t}$ (red curve, also shown in Figure 1) is estimated with equation (1). The impact threshold $u_{*, i t}$ is estimated with the saltation model COMSALT for three different values of the cohesion coefficient $\beta$ (blue curves), which span the whole uncertainty range of cohesive forces among organic grains on Titan. The settling velocity $w_{s}$ (green curve) is calculated by balancing the gravitational, drag, and buoyancy forces acting on spherical grains in still air. The shaded areas indicate one standard error from the mean.

\section{Size of saltating grains on Titan}

Our results have thus far indicated that the minimum fluid threshold corresponds to a particle size $d \approx 2$ $\mathrm{mm}$, whereas the minimum impact threshold corresponds to a particle size $d \approx 0.1 \mathrm{~mm}$. It follows that the size of grains in saltation may depend on the wind speed, that is, coarser near the transport initiation threshold and finer near to the transport cessation threshold.

To investigate the size range of saltating grains, we assume that Titan's surface presents mixed-sized grains in the range $0.05-2 \mathrm{~mm}$, similar to sand grains on Earth. We further assume that, whenever the wind speed exceeds the minimum fluid threshold, all surface grain sizes are susceptible to motion according to the equal susceptibility principle Martin and Kok, 2019. We follow a similar approach to previous studies Greeley and Iversen, 1985, Nishimura and Hunt, 2000, Sullivan and Kok, 2017 and investigate the size distribution of grains in saltation by evaluating the ratio $w_{s} / u_{*}$, where $w_{s}$ is the terminal fall velocity as a function of the 
grain size (green curve in Figure 3). Values of $w_{s} / u_{*}$ near unity indicate that gravitational and turbulent forces are of the same order of magnitude and grain transport is therefore transitional between saltation and suspension. We find that, near the threshold for transport initiation $\left(u_{*} \approx u_{*, f t}\right), w_{s} / u_{*}>1$ for $d>0.2$ $\mathrm{mm}$, whereas, near the threshold for transport cessation $\left(u_{*} \approx u_{*, i t}\right)$, the ratio $w_{s} / u_{*}>1$ for $d>0.05 \mathrm{~mm}$. These results suggest that the size of saltating grains at the onset of transport lies in the range $d \approx 0.2-2$ $\mathrm{mm}$, as smaller grains become suspended in turbulent eddies. Conversely, close to the cessation of transport, the size of saltating grains lies in the lower range $d \approx 0.05-0.1 \mathrm{~mm}$, because the wind speed is not sufficient to sustain saltation of larger grains through rebound and splash.

\section{Mass flux scaling on Titan}

Our analyses indicate that initiation and cessation of saltation on Titan occur at very different wind speeds, yielding a ratio between the impact and fluid thresholds $u_{*, i t} / u_{*, f t} \approx 0.25$ much smaller than previously thought Kok et al. 2012. This suggests that saltation on Titan can be sustained at much lower wind speeds than those required to initiate it, similarly to the transport mechanisms on Mars Sullivan and Kok, 2017]. We find that the surface wind speeds in Titan's equatorial band $\left(30^{\circ} \mathrm{S}-30^{\circ} \mathrm{N}\right)$ predicted by general circulation models Tokano, 2010, Lebonnois et al., 2012, Lora et al., 2015, Newman et al., 2016] exceed the impact threshold $15-30 \%$ of Titan's year and can therefore sustain sediment transport (see the supporting information section S5). To quantify the sediment transport rates driven by the prevailing circulation, we derive a saltation mass flux parameterization for Titan conditions and test its accuracy against COMSALT simulations.

Previous studies have suggested that the general expression for the steady-state saltation mass flux reads $Q=\rho_{f}\left(u_{*}^{2}-u_{u_{*}, i t}^{2}\right) L /\langle\Delta v\rangle$, where $L$ is the mean hop length of saltating grains and $\langle\Delta v\rangle$ is the mean difference in grain horizontal velocity before and after impacting the bed Durán et al., 2011, Kok et al. 2012. In steady-state saltation, the impact velocity is bound to yield a mean replacement capacity equal to 1 , that is, to generate on average one splashed grain for every impactor that fails to rebound Ungar and Haff 1987. It follows that $\langle\Delta v\rangle$ is independent of $u_{*}$ and rather scales as $\langle\Delta v\rangle \sim u_{*, i t}$. Conversely, the hop length $L$ is determined in part by particle speeds higher up in the saltation layer. For saltation on Earth, $L$ is only a weak function of $u_{*}$ and is often assumed to scale as $L \sim u_{*, i t}^{2} / g$ Durán et al. 2011, Martin and Kok, 2017. However, saltation on Titan is characterized by much longer hop times than on Earth due to the higher air density, thus higher air drag, and smaller gravity. It follows that particle speeds in the upper part of the saltation layer can scale with $u_{*}$ without producing a strong increase in impact velocity. Assuming similar proportions in the populations of grains in saltation and in reptation near the surface Andreotti, 2004, Lämmel et al., 2012, the mean hop length on Titan scales as $L \sim u_{*, i t}\left(u_{*}+u_{*, i t}\right) / g$. The proposed scalings for $L$ and $\langle\Delta v\rangle$ on Titan are confirmed by COMSALT simulations (see supporting information section S6) and yield a mass flux

$$
Q=A \frac{\rho_{f}}{g}\left(u_{*}^{2}-u_{*, i t}^{2}\right)\left(u_{*}+u_{*, i t}\right) \eta_{q}
$$

where $A \approx 2.3$ is a dimensionless scaling coefficient and $\eta_{q} \in(0,1)$ is the intermittency factor that quantifies the fraction of time that saltation is active when the unsteady wind speeds oscillate between the impact and fluid thresholds. We calculate $\eta_{q}$ using the parameterization of Comola et al. 2019b], which was validated using extensive field data from three different locations on Earth. This parameterization predicts transport intermittency based on the friction velocity and the Obukhov stability parameter, which quantify the shear-generated and buoyancy-generated turbulence driving the variability in wind speed (see supporting information section S5 for details). We find that the mass fluxes predicted with equation (6) are in good agreement with steady-state mass fluxes obtained with COMSALT for a variety of particle sizes and friction velocities (Figure 4a).

The mass flux scaling $Q \propto u_{*}^{3}$ of equation (6) is typical of particle flows that dissipate energy through a 
combination of fluid drag, particle-bed collisions, and binary collisions between airborne grains Pähtz and Durán, 2020 and is found in another mass flux parameterization by Kawamura 1951], which has been commonly used in planetary saltation studies [e.g., White, 1979, Lee and Thomas, 1995, Charnay et al. 2015, Gebhardt et al. 2020]. However, in the original parameterization by Kawamura [1951] it is assumed that fluid lifting drives continuous sediment transport and that the friction velocity at the bed, for which the threshold friction velocity is a proxy in the mass flux equation, is equal to the fluid threshold. We find that our parameterization that accounts for transport intermittency (equation (6)) predicts a significantly larger mass flux than the continuous transport parameterization by Kawamura [1951] when the wind speed lies between the impact and fluid thresholds, as is often the case on Titan (Figure 4b).
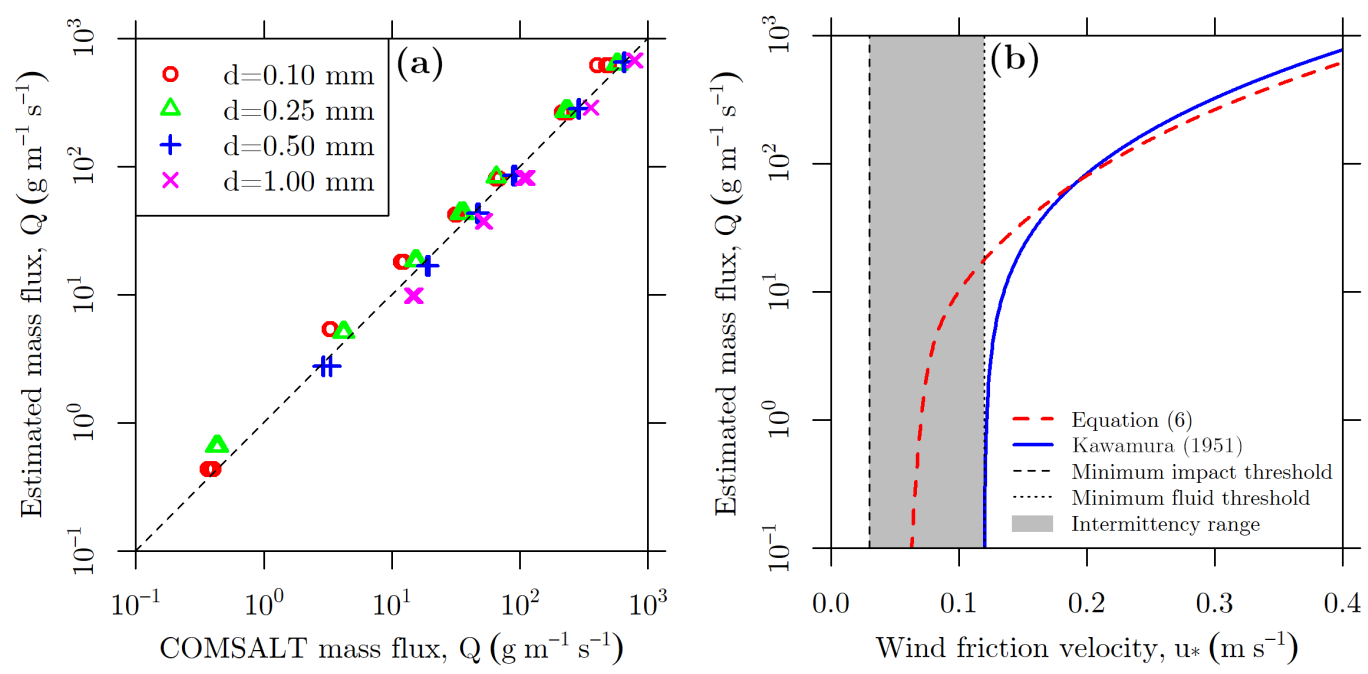

Figure 4: Saltation mass flux scaling for Titan conditions. (a) Comparison of mass fluxes predicted by COMSALT and estimated with equation (6) for different combinations of particle size and wind friction velocity. Because COMSALT simulates continuous transport, we assumed $\eta_{q}=1$ in equation (6). (b) Titan mass flux scaling predicted with equation (6) in conditions of intermittent transport $\left(\eta_{q}<1\right.$, dashed red line) and with the mass flux equation by Kawamura 1951 commonly used in planetary aeolian transport studies (solid blue line). We assumed that grain sizes on Titan lie within the range $0.05-2 \mathrm{~mm}$, similar to sand on Earth, and set the impact and fluid thresholds equal to the corresponding minima in this range, that is $u_{*, i t}=0.03 \mathrm{~m} \mathrm{~s}^{-1}$ (dashed black line) and $u_{*, f t}=0.12 \mathrm{~m} \mathrm{~s}^{-1}$ (dotted black line). The gray area indicates the range of saltation intermittency between the impact and fluid thresholds. We computed the intermittency factor $\eta_{q}$ assuming a neutrally stable atmosphere and a Titan boundary layer height equal to $3 \mathrm{~km}$ Lorenz et al. 2010. (see supporting information for details on the calculation of $\eta_{q}$ ).

\section{Aeolian activity on Titan}

We assess the aeolian transport potential of Titan's general circulation by implementing the proposed mass flux parameterization (equation (6) in the Titan Atmospheric Model (TAM) Lora et al. 2015, 2019 accounting for the effect of large-scale topography Corlies et al., 2017] (see supporting information section $\mathrm{S} 7$ for additional detail). We perform runtime calculations of the wind-driven saltation mass flux for five Titan years, using surface friction velocities and intermittency factors computed at the model time step of 10 minutes, horizontal resolutions of approximately 5.6 degrees, and a vertical grid with 48 levels of varying pressure thickness. We assign the instantaneous mass flux direction equal to the corresponding wind direction at the first node above the surface.

The simulated yearly mass fluxes on Titan show a significant spatial variability in magnitude and direction 
(red arrows in Figure 5a). We find that accounting for Titan's large-scale topography leads to drift directions that diverge significantly from Titan's prevailing easterly circulation (the drift directions in absence of topography are shown in Figure S7 of the supporting information). The effect of large-scale topography might thus partly explain the inconsistency between the direction of the prevailing winds and the eastward orientation of the linear dunes, which previous studies have thus far attributed to the occurrence of eastward-propagating methane storms [Charnay et al., 2015, long climate cycles [Ewing et al., 2015], and orbital forcing [McDonald et al. 2016. The model results indicate that Titan presents regions of significant aeolian activity, with yearly mass fluxes of the order of $10^{5} \mathrm{~kg} \mathrm{~m}^{-1}$ year ${ }^{-1}$ (note that one Titan year corresponds to approximately 29.5 Earth years). Furthermore, sediment transport is active approximately $30 \%$ of the year (Figure $5 \mathrm{~b}$ ), with higher saltation activity during the summer season in the northern and southern regions (blue and red lines in Figure 5b) and with little seasonality in the equatorial region (green line in Figure 5b).
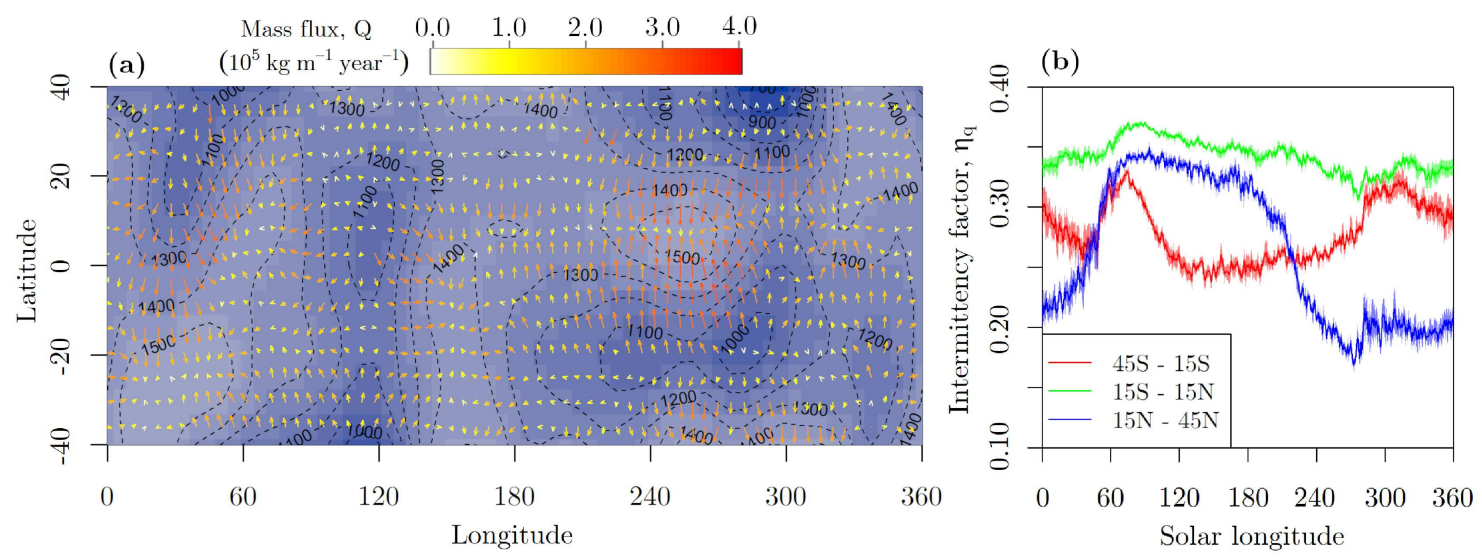

Figure 5: Sediment transport rates and intermittency on Titan. (a) Cumulated mass fluxes (yellow and red arrows) predicted by Titan general circulation model TAM using equation (6) for one Titan year (approximately 29.5 Earth years). Dashed black lines and background blue colors indicate surface elevation at the model resolution. (b) Intermittency factor annual variability in the equatorial region (green line), southern region (red line), and northern region (blue line). Higher values of $\eta_{q}$ indicate more intense saltation activity. The shaded areas indicate one standard error from the mean.

\section{Discussion}

We combined experimental results, theory, and modeling to investigate the conditions that lead to sediment transport initiation and cessation on Titan. We found that the minimum fluid threshold $\left(u_{*} \approx 0.12 \mathrm{~m} \mathrm{~s}^{-1}\right)$ corresponds to a particle size $d \approx 2 \mathrm{~mm}$, whereas the minimum impact threshold $\left(u_{*} \approx 0.03 \mathrm{~m} \mathrm{~s}^{-1}\right)$ corresponds to a particle size $d \approx 0.1 \mathrm{~mm}$ (Figure 3 ). Furthermore, the impact threshold is smaller than the fluid threshold for grains smaller than $2 \mathrm{~mm}$, whereas the fluid threshold is smaller than the impact threshold for larger grains. The granular splash process is thus more effective than aerodynamic forces in lifting submillimeter grains from the surface. Conversely, transport of super-millimeter grains is primarily sustained by aerodynamic entrainment, which typically occurs in dense fluid flows such as fluvial environments on Earth Pähtz et al. 2020. It is noteworthy that the fluid threshold values predicted by equation (1) are representative of wind tunnel conditions, where turbulence scales are much smaller than in the atmospheric boundary layer. Because the aerodynamic entrainment is predominantly caused by turbulent fluctuation events Pähtz et al. 2020, it is possible that the fluid threshold on Titan may be up to $50 \%$ smaller than what is predicted by equation (1) due to the large turbulent motions in the thick boundary layer Pähtz et al., 2018. Despite these uncertainties, the separation between the minimum fluid and impact thresholds on Titan is likely to be significantly larger than on Earth Martin and Kok, 2018, Ho et al., 2011]. Much like saltation on Mars, Titan saltation may therefore be characterized by a process of hysteresis whereby 
the occurrence of transport below the fluid threshold depends on the history of the wind, that is, saltation occurs only if transport was initiated $\left(u_{*}>u_{*, f t}\right)$ more recently than it was terminated $\left(u_{*}<u_{*, i t}\right)$ [e.g., Kok, 2010a.

We investigated the size of saltating grains on Titan by evaluating the ratio between settling velocity and friction velocity, $w_{s} / u_{*}$, for a wide range of grain sizes. We found that the size range of saltating grains may depend on the wind speed, varying from $0.2-2 \mathrm{~mm}$ near the transport initiation threshold to $0.05-0.1 \mathrm{~mm}$ near the transport cessation threshold. Note that our analysis based on the equal susceptibility assumption may provide incorrect estimations of the size of saltating grains if some grain sizes are more susceptible to motion than others. For instance, Sullivan and Kok 2017 have found that $0.1 \mathrm{~mm}$ grains are prevalent in actively-migrating ripples on Mars even though $w_{s} / u_{*, f t}$ is much larger than one for this particle size.

Our analyses further indicated that the saltation mass flux on Titan scales with the third power of the wind friction velocity, that is, $Q \propto u_{*}^{3}$ (equation (6) and Figure 4). This suggests a higher sensitivity of the transport rate to the wind speed compared to Earth conditions, where $Q \propto u_{*}^{2}$ Martin and Kok, 2017. However, the larger separation between the fluid and impact thresholds on Titan, combined with the typically low wind speeds of the prevailing circulation, is more likely to cause intermittent transport than on Earth Comola et al. 2019b. We implemented the proposed mass flux scaling in the Titan general circulation model TAM and estimated that the regions with more intense aeolian activity present transport rates of the order of $10^{5} \mathrm{~kg} \mathrm{~m}^{-1}$ per Titan year (Figure 5a). This is similar to the transport rate observed on the most mobile dune fields on Mars [see, e.g., Bridges et al., 2012, where the atmosphere is more energetic but less dense than on Titan. Our TAM simulations indicate that transport intermittency causes saltation to be active approximately $30 \%$ of the year, with significant seasonal variations (Figure 5b). Given the poor constraints on Titan's topography and the limitations involved in solving for convective processes in current Titan GCMs, large uncertainties remain in how methane storms and fine-scale topographic features affect Titan's aeolian activity. Nevertheless, our analyses indicated that Titan's prevailing winds are capable of generating a significant "background" aeolian activity and that the effect of large-scale topography on near-surface winds is critical to explaining Titan's geomorphology and landscape evolution.

\section{Acknowledgments}

This research was supported by the Swiss National Science Foundation (project number P2ELP2_178219). X. $\mathrm{Yu}$ is supported by the 51 Pegasi b postdoctoral fellowship from the Heising-Simons Foundation. Additional support was provided by NASA Outer Planets Research grant NNX14AR23G to J. F. Kok. The authors thank Tetsuya Tokano, Claire Newman, Kirby Runyon, and Benjamin Charnay for sharing their Titan GCM and RCM model outputs. The authors also wish to thank Thomas Pähtz for the insightful discussions on the uncertainty in the fluid threshold value and the effect of the viscous sublayer on the impact threshold. All data presented in this paper will be made available at the following repository doi:10.17632/97j874sph6.1.

\section{References}

M. Ammi, L. Oger, D. Beladjine, and A. Valance. Three-dimensional analysis of the collision process of a bead on a granular packing. Phys. Rev. E, 79(2):021305, 2009.

R. S. Anderson and P. K. Haff. Wind modification and bed response during saltation of sand in air. In Aeolian Grain Transport 1, pages 21-51. Springer, 1991.

B. Andreotti. A two-species model of aeolian sand transport. J. Fluid Mech., 510:47-70, 2004.

R. A. Bagnold. The transport of sand by wind. Geogr. J., 89(5):409-438, 1937.

N. T. Bridges, F. Ayoub, J. P. Avouac, S. Leprince, A. Lucas, and S. Mattson. Earth-like sand fluxes on Mars. Nature, 485(7398):339-342, 2012. 
D. M. Burr, N. T. Bridges, J. R. Marshall, J. K. Smith, B. R. White, and J. P. Emery. Higher-than-predicted saltation threshold wind speeds on Titan. Nature, 517(7532):60-63, 2015.

B. Charnay, E. Barth, S. Rafkin, C. Narteau, S. Lebonnois, S. Rodriguez, S. C. Du Pont, and A. Lucas. Methane storms as a driver of Titan's dune orientation. Nat. Geosci., 8(5):362-366, 2015.

WS Chepil. Dynamics of wind erosion: II. Initiation of soil movement. Soil Sci., 60(5):397, 1945.

F. Comola and M. Lehning. Energy- and momentum-conserving model of splash entrainment in sand and snow saltation. Geophys. Res. Lett., 44(3):1601-1609, 2017.

F. Comola, J. Gaume, J. F. Kok, and M. Lehning. Cohesion-induced enhancement of aeolian saltation. Geophys. Res. Lett., 46(10):5566-5574, 2019a.

F Comola, J. F. Kok, M. Chamecki, and R. L. Martin. The intermittency of wind-driven sand transport. Geophys. Res. Lett., 2019b.

P. Corlies, A. G. Hayes, S. P. D. Birch, R. Lorenz, B. W. Stiles, R. Kirk, V. Poggiali, H. Zebker, and L. Iess. Titan's topography and shape at the end of the Cassini mission. Geophys. Res. Lett., 44(23):11-754, 2017.

M. Corn. The adhesion of solid particles to solid surfaces, I. A review. J Air Pollut. Control Assoc., 11(11): 523-528, 1961.

J. Crassous, D. Beladjine, and A. Valance. Impact of a projectile on a granular medium described by a collision model. Phys. Rev. Lett., 99(24):248001, 2007.

O. Durán, P. Claudin, and B. Andreotti. On aeolian transport: Grain-scale interactions, dynamical mechanisms and scaling laws. Aeolian Res., 3(3):243-270, 2011.

R. C. Ewing, A. G. Hayes, and A. Lucas. Sand dune patterns on Titan controlled by long-term climate cycles. Nat. Geosci., 8(1):15-19, 2015.

B Fletcher. The incipient motion of granular materials. J. Phys. D, 9(17):2471, 1976.

C. Gebhardt, A. Abuelgasim, R. M. Fonseca, J. Martín-Torres, and M-P Zorzano. Fully interactive and refined resolution simulations of the Martian dust cycle by the MarsWRF model. J. Geophys. Res., 125 (9):e2019JE006253, 2020.

M. Gordon and C. McKenna-Neuman. A study of particle splash on developing ripple forms for two bed materials. Geomorphology, 129(1-2):79-91, 2011.

R. Greeley and J. D. Iversen. Wind as a geological process: on Earth, Mars, Venus and Titan. Cambridge University Press, New York, 1985.

C. He, S. M. Hörst, S. Riemer, J. A. Sebree, N. Pauley, and V. Vuitton. Carbon monoxide affecting planetary atmospheric chemistry. Astrophys. J. Lett., 841(2):L31, 2017.

T. D. Ho, A. Valance, P. Dupont, and A. Ould El Moctar. Scaling laws in Aeolian sand transport. Phys. Rev. Lett., 106(9):094501, 2011.

S. M. Hörst and M. A. Tolbert. In situ measurements of the size and density of Titan aerosol analogs. Astrophys. J. Lett., 770(1):L10, 2013.

H. Imanaka, D. P. Cruikshank, B. N. Khare, and C. P. McKay. Optical constants of Titan tholins at midinfrared wavelengths $(2.5-25 \mu \mathrm{m})$ and the possible chemical nature of Titan's haze particles. Icarus, 218 (1):247-261, 2012.

J. N. Israelachvili. Intermolecular and Surface Forces. Wiley Online Library, 1986.

J. D. Iversen, J. B. Pollack, R. Greeley, and B. R. White. Saltation threshold on Mars: The effect of interparticle force, surface roughness, and low atmospheric density. Icarus, 29(3):381-393, 1976. 
R. Kawamura. Study on sand movement by wind. Rept. Inst. Sci. Technol., 5:95-112, 1951.

J. F. Kok. Difference in the wind speeds required for initiation versus continuation of sand transport on Mars: Implications for dunes and dust storms. Phys. Rev. Lett., 104(7):074502, 2010a.

J. F. Kok. An improved parameterization of wind-blown sand flux on Mars that includes the effect of hysteresis. Geophys. Res. Lett., 37(12), 2010b.

J. F. Kok and N. O. Renno. A comprehensive numerical model of steady state saltation (COMSALT). J. Geophys. Res., 114(D17), 2009.

J. F. Kok, E. J. R. Parteli, T. I. Michaels, and D. B. Karam. The physics of wind-blown sand and dust. Rep. Prog. Phys., 75(10):106901, 2012.

M. Lämmel, D. Rings, and K. Kroy. A two-species continuum model for aeolian sand transport. New J. Phys., 14(9):093037, 2012.

S. Lebonnois, J. Burgalat, P. Rannou, and B. Charnay. Titan global climate model: A new 3-dimensional version of the IPSL Titan GCM. Icarus, 218(1):707-722, 2012.

P. Lee and P. C. Thomas. Longitudinal dunes on Mars: Relation to current wind regimes. J. Geophys. Res., 100(E3):5381-5395, 1995.

R. M. C. Lopes, S. D. Wall, C. Elachi, S. P. D. Birch, P. Corlies, A. Coustenis, A. G. Hayes, J. D. Hofgartner, M. A. Janssen, R. L. Kirk, et al. Titan as revealed by the Cassini radar. Space Sci. Rev., 215(4):1-50, 2019.

J. M. Lora, J. I. Lunine, and J. L. Russell. GCM simulations of Titan's middle and lower atmosphere and comparison to observations. Icarus, 250:516-528, 2015.

J. M. Lora, T. Tokano, J. V. d'Ollone, S. Lebonnois, and R. D. Lorenz. A model intercomparison of Titan's climate and low-latitude environment. Icarus, 333:113-126, 2019.

R. D. Lorenz. Physics of saltation and sand transport on Titan: A brief review. Icarus, 230:162-167, 2014.

R. D. Lorenz, P. Claudin, B. Andreotti, J. Radebaugh, and T. Tokano. A $3 \mathrm{~km}$ atmospheric boundary layer on Titan indicated by dune spacing and Huygens data. Icarus, 205(2):719-721, 2010.

R.D. Lorenz, S. Wall, J. Radebaugh, G. Boubin, E. Reffet, M. Janssen, E. Stofan, R. Lopes, R. Kirk, C. Elachi, et al. The sand seas of Titan: Cassini RADAR observations of longitudinal dunes. Science, 312 (5774):724-727, 2006.

S. M. MacKenzie, S. P. D. Birch, S. M. Hörst, C. Sotin, E. Barth, J. M. Lora, M. G. Trainer, P. Corlies, M. J. Malaska, E. Sciamma-O'Brien, et al. Titan: Earth-like on the outside, ocean world on the inside. arXiv preprint arXiv:2102.08472, 2021.

R. L Martin and J. F. Kok. Wind-invariant saltation heights imply linear scaling of aeolian saltation flux with shear stress. Sci. Adv., 3(6):e1602569, 2017.

R. L. Martin and J. F. Kok. Distinct thresholds for the initiation and cessation of aeolian saltation from field measurements. J. Geophys. Res., 123(7):1546-1565, 2018.

R. L. Martin and J. F. Kok. Size-independent susceptibility to transport in aeolian saltation. J. Geophys. Res., 124(7):1658-1674, 2019.

G. D. McDonald, A. G. Hayes, R. C. Ewing, J. M. Lora, C. E. Newman, T. Tokano, A. Lucas, A. Soto, and G. Chen. Variations in Titan's dune orientations as a result of orbital forcing. Icarus, 270:197-210, 2016.

J. S. Méndez-Harper, G. D. McDonald, J. Dufek, M. J. Malaska, D. M Burr, A. G. Hayes, J. McAdams, and J. J. Wray. Electrification of sand on Titan and its influence on sediment transport. Nat. Geosci., 10(4): 260-265, 2017. 
P. Nalpanis, J. C. R. Hunt, and C. F. Barrett. Saltating particles over flat beds. J. Fluid Mech., 251:661-685, 1993.

C. E. Newman, M. I. Richardson, Y. Lian, and C. Lee. Simulating Titan's methane cycle with the TitanWRF general circulation model. Icarus, 267:106-134, 2016.

K. Nishimura and J. C. R. Hunt. Saltation and incipient suspension above a flat particle bed below a turbulent boundary layer. J. Fluid Mech., 417:77-102, 2000.

T. Pähtz and O. Durán. The cessation threshold of nonsuspended sediment transport across aeolian and fluvial environments. J. Geophys. Res., 123(8):1638-1666, 2018.

T. Pähtz and O. Durán. Unification of aeolian and fluvial sediment transport rate from granular physics. Phys. Rev. Lett., 124(16):168001, 2020.

T. Pähtz, M. Valyrakis, X. Zhao, and Z. Li. The critical role of the boundary layer thickness for the initiation of aeolian sediment transport. Geosciences, 8(9):314, 2018.

T. Pähtz, A. H. Clark, M. Valyrakis, and O. Durán. The physics of sediment transport initiation, cessation, and entrainment across aeolian and fluvial environments. Rev. Geophys., 58(1):e2019RG000679, 2020.

T. Pähtz, Y. Liu, Y. Xia, P. Hu, Z. He, and K. Tholen. Unified model of sediment transport threshold and rate across weak and intense subaqueous bedload, windblown sand, and windblown snow. J. Geophys. Res., page e2020JF005859, 2021.

J. Radebaugh, R. D. Lorenz, J. I. Lunine, S. D. Wall, G. Boubin, E. Reffet, R. L. Kirk, R. M. Lopes, E. R. Stofan, L. Soderblom, et al. Dunes on Titan observed by Cassini RADAR. Icarus, 194(2):690-703, 2008.

J. Radebaugh, R. Lorenz, T. Farr, P. Paillou, C. Savage, and C. Spencer. Linear dunes on Titan and Earth: Initial remote sensing comparisons. Geomorphology, 121(1-2):122-132, 2010.

M. A. Rice, B. B. Willetts, and I. K. McEwan. An experimental study of multiple grain-size ejecta produced by collisions of saltating grains with a flat bed. Sedimentology, 42(4):695-706, 1995.

M. A. Rice, B. B. Willetts, and I. K. McEwan. Observations of collisions of saltating grains with a granular bed from high-speed cine-film. Sedimentology, 43(1):21-31, 1996.

S. Rodriguez, S. Le Mouélic, J. W. Barnes, J. F. Kok, S. C. R. Rafkin, R. D. Lorenz, B. Charnay, J. Radebaugh, C. Narteau, T. Cornet, et al. Observational evidence for active dust storms on Titan at equinox. Nat. Geosci., 11(10):727-732, 2018.

Y. Shao and H. Lu. A simple expression for wind erosion threshold friction velocity. J. Geophys. Res., 105 (D17):22437-22443, 2000.

R. Sullivan and J. F. Kok. Aeolian saltation on Mars at low wind speeds. J. Geophys. Res., 122(10): 2111-2143, 2017.

T. Tokano. Dune-forming winds on Titan and the influence of topography. Icarus, 194(1):243-262, 2008.

T. Tokano. Relevance of fast westerlies at equinox for the eastward elongation of Titan's dunes. Aeolian Res., 2(2-3):113-127, 2010.

J. E. Ungar and P. K. Haff. Steady state saltation in air. Sedimentology, 34(2):289-299, 1987.

B. R. White. Soil transport by winds on Mars. J. Geophys. Res., 84(B9):4643-4651, 1979.

B. B. Willetts and M. A. Rice. Inter-saltation collisions. In Proc. Int. Workshop on Physics of Blown Sand (Aarhus, Denmark), volume 1, pages 83-100, 1985.

B. B. Willetts and M. A. Rice. Collisions in aeolian saltation. Acta Mech., 63(1-4):255-265, 1986. 
B. B. Willetts and M. A. Rice. Collisions of quartz grains with a sand bed: the influence of incident angle. Earth Surf. Process. Landf., 14(8):719-730, 1989.

X Yu, S M Hörst, C He, P McGuiggan, and N T Bridges. Direct measurement of interparticle forces of Titan aerosol analogs ("tholin") using atomic force microscopy. J. Geophys. Res., 122(12):2610-2622, 2017.

X. Yu, S. M. Hörst, C. He, and P. McGuiggan. Single particle triboelectrification of Titan sand analogs. Earth Planet. Sc. Lett., 530:115996, 2020a.

X. Yu, S. M. Hörst, C. He, P. McGuiggan, K. Kristiansen, and X. Zhang. Surface energy of the Titan aerosol analog "tholin". Astrophys. J., 905(2):88, 2020b.

AW Zingg. Wind tunnel studies of the movement of sedimentary material. In Proc. 5th Hydraulics Conference Bulletin, volume 34, pages 111-135. Inst. of Hydraulics Iowa City, 1953. 